\title{
Direct multipulse laser processing of titanium oxide - graphene oxide nanocomposite thin films
}

\author{
A. Pérez del Pino, ${ }^{1}$ A. Datcu, ${ }^{2}$ E. György ${ }^{1,2 *}$ \\ ${ }^{1}$ Consejo Superior de Investigaciones Científicas, Instituto de Ciencia de Materiales de \\ Barcelona (CSIC-ICMAB), Campus UAB, 08193 Bellaterra, Spain \\ ${ }^{2}$ National Institute for Lasers, Plasma and Radiation Physics, P. O. Box MG 36, 76900 \\ Bucharest V, Romania
}

\begin{abstract}
Nanocomposite thin films consisting of titanium oxide $\left(\mathrm{TiO}_{2}\right)$ nanoparticles (NPs) and graphene oxide (GO) platelets were deposited by spin-coating technique. The obtained films were submitted to direct laser irradiation using a frequency quadrupled Nd:YAG $(\lambda=266 \mathrm{~nm}$, $\tau_{\mathrm{FWHM}} \cong 3 \mathrm{~ns}, v=10 \mathrm{~Hz}$ ) laser source. The effect of the laser processing conditions, as laser fluence value and number of subsequent laser pulses incident onto the same target location, on the surface morphology, crystalline structure, and chemical composition of the $\mathrm{TiO}_{2} / \mathrm{GO}$ nanocomposite thin films was systematically investigated. The laser fluence values were maintained below the vaporisation threshold of the irradiated composite material. With the increase of the laser fluence and number of incident laser pulses melting and coalescence of the $\mathrm{TiO}_{2}$ NPs into inter-connected aggregates as well as rippling of the GO platelets take place. The gradual reduction of GO platelets and the onset of anatase to rutile phase transition were observed at high laser fluence values.
\end{abstract}

Keywords: titanium oxide - graphene oxide nanocomposite thin films; direct laser irradiation 


\section{Introduction}

Titanium dioxide $\left(\mathrm{TiO}_{2}\right)$ is widely investigated due to its unique properties as high optical transmittance in the visible and IR spectral regions, high refractive index, good corrosion resistance, high hardness, nontoxicity, chemical as well as mechanical stability [1]. During the last years, a number of effective approaches have been developed to further improve the functional properties of $\mathrm{TiO}_{2}$, through modification with metal and nonmetal elements and structures, surface sensitization by dye molecules, as well as coupling with other semiconductor or carbon-based materials [2]. Among these, creation of hybrid composites through the addition of carbon nanomaterials represents one of the most promising approaches, largely motivated by the unique functional properties of carbon nanostructures. Indeed, carbon-based structures are key building blocks for many novel technological applications. In particular, graphene, a two-dimensional single atomic layer of $\mathrm{sp}^{2}$ bonded carbon atoms [3], is a promising material because of its unique and exceptional electrical [3, 4], thermal [5], and optical properties [6]. Graphene oxide (GO), a graphene derivative with oxygen groups on the carbon bone scaffold of graphene, is also a widely investigated valuable novel material [7, 8]. GO exhibits outstanding functional properties such as hydrophilicity [9], mechanical strength [10, 11], and chemical stability [12]. Moreover, through an accurate control of the O/C ratio, tuning of the functional properties can be achieved. Fully oxidized GO is an insulator and hydrophilic while partially reduced GO (rGO) has semiconducting properties with band gap determined by its $\mathrm{O} / \mathrm{C}$ ratio [13]. The hydrophilic to hydrophobic transition process is also governed by the amount of oxygen containing functional groups of GO $[13,14]$. As known, chemical reduction is the most used method to obtain rGO $[15,16]$. However, most reducing agents, such as hydrazine hydrate [15] or sodium borohydrate [16] are toxic and/or explosive. Thus, continuous endeavors are directed towards the development and optimization of eco-friendly reducing methods, ensuring an accurate control over the $\mathrm{O} / \mathrm{C}$ ratio of the obtained rGO.

In form of surface coatings, $\mathrm{TiO}_{2} / \mathrm{GO}$ and $\mathrm{TiO}_{2} / \mathrm{rGO}$ hybrid composites are of great scientific interest for various applications such as photocatalytic decomposition of organic pollutants, generation of hydrogen gas through water splitting, self-cleaning surfaces, $\mathrm{CO}_{2}$ reduction, gas sensors, solar cells, laser diodes, photodetectors, or energy-harvesting devices [17-19]. Our previous investigations revealed that both photocatalytic efficiency as well as surface wetting properties of $\mathrm{TiO}_{2}$ / GO composite layers deposited by laser-based technique 
are improved as compared to the pure $\mathrm{TiO}_{2}$ counterparts [20, 21]. Moreover, direct laser irradiation of drop-casted $\mathrm{ZnO} / \mathrm{GO}$ composites in nitrogen atmosphere lead to doping of rGO and to the reduction of the water contact angle, the surface becoming super-hydrophilic under UV light irradiation [22]. In addition, one of the major requirements for the implementation of the layers in all mentioned application fields is their good adhesion to the underlying surface. Our previous results on laser irradiation of drop-casted $\mathrm{ZnO} / \mathrm{GO}$ composite films showed that both their stability as well as adhesion properties improved after laser treatment [22].

In the present work we investigated the effect of UV laser irradiation on the surface morphology, crystalline structure, and chemical composition of $\mathrm{TiO}_{2}$ / GO nanocomposite layers previously deposited by spin-coating, using $\mathrm{TiO}_{2} / \mathrm{GO}$ aqueous solutions. The laser irradiations experiments were performed in vacuum. The dependence of the surface morphology, crystalline structure, and chemical composition of the layers on the laser parameters as incident laser fluence and number of subsequent laser pulses was investigated. Our results showed that controlled and gradual reduction of the GO platelets in the $\mathrm{TiO}_{2}$ / GO hybrid composites can be obtained through eco-friendly direct laser irradiation technique whereas the stoichiometry of the $\mathrm{TiO}_{2} \mathrm{NPs}$ remains unaltered.

\section{Experimental}

Dispersions consisting of 1 wt.\% anatase phase $\mathrm{TiO}_{2}$ nanoparticles (Aldrich, 99.5\%) and 0.5 wt. \% GO platelets (NanoInnova Technologies,Madrid, Spain) in distilled water were prepared for the deposition of the initial $\mathrm{TiO}_{2} / \mathrm{GO}$ composite thin films by spin-coating technique. For the deposition of each film $50 \mu \mathrm{l}$ dispersions were dropped on the surface of the substrates in $5 \times 10 \mu$ steps. The substrates consisted of $10 \times 10 \mathrm{~mm}^{2}(001) \mathrm{SiO}_{2}$ quartz plates. The rotational frequency of the substrates was fixed at $800 \mathrm{rpm}$. Each deposition was performed by spinning the substrates during $1 \mathrm{~s}$. After deposition the samples were dried in air at normal atmospheric conditions. The laser irradiation experiments were performed in a vacuum chamber, evacuated down to a residual pressure of $10^{-4} \mathrm{~Pa}$. A Nd:YAG Qantel Brilliant B $\left(\lambda=266 \mathrm{~nm}, \tau_{\mathrm{FWHM}}=3 \mathrm{~ns}, v=10 \mathrm{~Hz}\right)$ laser source was used for the laser irradiation experiments. The number of subsequent laser pulses was chosen in the range (100 - 1000). The incident laser fluence on the surface of the samples was set at values in the range 
(20 - 80) $\mathrm{J} / \mathrm{cm}^{2}$. A non-irradiated $\mathrm{TiO}_{2}$ / GO composite thin film obtained by spin-coating, using the same experimental conditions as for the samples submitted to laser irradiation, was used as reference.

The surface morphology of the reference and laser irradiated thin films was investigated by field emission scanning electron microscopy (FE-SEM) with the aid of a QUANTA FEI 200 FEG-ESEM system. The micro-Raman measurements were performed in backscattering configuration, with a Jobin-Yvon T6400 spectrometer equipped with an optical microscope. For excitation, the $532 \mathrm{~nm}$ laser beam was focused with a 50x objective over a $2 \mu \mathrm{m}$ diameter spot area on the sample surface. The laser power on the sample was $0.8 \mathrm{~mW}$, avoiding thermal heating during the measurements. Three spectra with $10 \mathrm{~s}$ integration time were registered for each analised surface area.

\section{Results and discussion}

The FE-SEM image of the non-irradiated $\mathrm{TiO}_{2} / \mathrm{GO}$ nanocomposite thin film obtained by spin coating technique from a solution containing 1 wt.\% $\mathrm{TiO}_{2}$ NPs and $0.5 \mathrm{wt} . \%$ GO platelets in distilled water is shown in Fig. 1. As can be observed, the GO platelets are partially covered by $\mathrm{TiO}_{2} \mathrm{NPs}$. The $\mathrm{TiO}_{2}$ NPs have a homogeneous size distribution with an average diameter around $20 \mathrm{~nm}$.

The spin-coated $\mathrm{TiO}_{2} / \mathrm{GO}$ thin films were submitted to laser irradiation in vacuum at a residual pressure of $10^{-4} \mathrm{~Pa}$. At the lowest laser fluence value $20 \mathrm{~mJ} / \mathrm{cm}^{2}$, no significant changes of the surface morphology were observed in the studied range (100-1000) of number of subsequent laser pulses. At 1000 subsequent laser pulses (Fig. 2a) the surface morphology is similar to that of the reference non-irradiated sample (Fig. 1). Indeed, the single pulse melting threshold of $\mathrm{TiO}_{2}$ thin films processed by an $\mathrm{UV} \mathrm{KrF}^{*}$ excimer laser source $(\lambda=248$ $\mathrm{nm}, \tau_{\mathrm{FWHM}}=25 \mathrm{~ns}$ ) was reported to be around $0.25 \mathrm{~J} / \mathrm{cm}^{2}$, corresponding to a laser intensity value of $10 \mathrm{MW} / \mathrm{cm}^{2}$ [23], well above our lowest, $20 \mathrm{~mJ} / \mathrm{cm}^{2}$ laser fluence value, equivalent to a laser intensity value of $6.6 \mathrm{MW} / \mathrm{cm}^{2}$. However, at the same number of pulses, the increase of the laser fluence to $40 \mathrm{~mJ} / \mathrm{cm}^{2}\left(13.3 \mathrm{MW} / \mathrm{cm}^{2}\right)$ leads to the onset of local melting and coalescence of $\mathrm{TiO}_{2}$ NPs (Fig. 2b). With the further increase of the laser fluence to 60 and 80 
$\mathrm{mJ} / \mathrm{cm}^{2}$ the molten NPs were found to merge into inter-connected aggregates, leading to the formation of a network-like structure (Fig. 2c,d). At this high fluence values the melting and coalescence of the $\mathrm{TiO}_{2}$ NPs takes place even at low, 100 number of subsequent laser pulses (Fig. 3a,b). We would like to note that at the same number of pulses, but lower, $40 \mathrm{~mJ} / \mathrm{cm}^{2}$ laser fluence values the irradiated surface morphology is identical to that of the reference nonirradiated sample (Fig. 1). However, with the further accumulation of the laser pulses, surface melting and coalescence of the molten particles takes place (Fig. 2b). As known, laserinduced melting threshold is reduced under multi-pulse irradiation conditions [22, 24-28]. This phenomenon, can be explained by enhanced absorption on existing defect sites [26-28], lattice defects or dislocations on the surface of the nanoparticles. Moreover, the creation of radiation-induced defects, frequently denoted in the scientific literature as incubation centers, are local alterations of the crystalline structure and changes in the chemical composition (metallization) owing that the experiments were carried out in high vacuum, enhances the absorption of the incident laser radiation [26-28]. Successive laser pulses increase the number of defects leading to the formation of new absorption bands and thereby enhance the absorptivity within the irradiated volume. The increase in the laser energy absorption, known in the literature as incubation effect, leads to the diminishment of the melting threshold under multipulse laser irradiation [27, 28].

Moreover, wrinkles and resonant ripple-like surface features formation on the surface of the GO sheets can be observed at the highest, $80 \mathrm{~mJ} / \mathrm{cm}^{2}$ laser fluence value (Fig. 3c). Similar surface features were observed on the surface of GO platelets in case of pure GO [29] as well as $\mathrm{ZnO} / \mathrm{GO}$ [22] and $\mathrm{Fe}_{3} \mathrm{O}_{4}$ / GO [29] composite films irradiated with the fourth-harmonic of a Nd:YAG laser, identical to that used in the experiments described in this work. Until certain extent similar surface modifications were observed in case of multiwall carbon nanotubes submitted to UV laser irradiation [30]. A stress driven process was suggested for wrinkle formation during repetitive heating and cooling down of graphene layers from temperatures of 1100-1300 K to room temperature [31]. The first wrinkles were found to appear after a cool down of around $400 \mathrm{~K}$. Upon heating and cooling cycles a hysteresis was observed in the appearance and disappearance of the wrinkles. Moreover, wrinkle patterns on the same surface location were similar during repeated heating and cooling cycles. This feature suggests that wrinkles nucleate at pre-existing features, defects of carbon rings, inducing local stress in the graphene lattice [31]. Under the action of the laser pulses, the creation of additional structural defects and variation of the chemical composition of GO platelets takes 
place. The locations with high enough density of defects would probably act as preferential absorption sites leading to local heating with the further accumulation of the laser pulses. The developed temperature gradients could contribute to stress-induced bending and the formation of wrinkles and ripple-like resonant surface features [31-33].

The micro-Raman spectra of the reference and laser irradiated $\mathrm{TiO}_{2}$ / GO composite thin films are presented in Fig. 4. The spectrum of the reference $\mathrm{TiO}_{2} / \mathrm{GO}$ film is composed by the lines situated at 145, 199, 397, 513, 519, and $635 \mathrm{~cm}^{-1}$ corresponding to the $\mathrm{Eg}_{(1)}, \mathrm{Eg}_{(2)}$, B1g $(1), A 1 g+B 1 g_{(2)}$, and $\mathrm{Eg}_{(3)}$ Raman vibrational modes of anatase phase $\mathrm{TiO}_{2}[34,35]$ as well as the lines at 1348 and $1594 \mathrm{~cm}^{-1}$ belonging to the well known D and G vibrational modes of carbon materials [36]. The $G$ mode is related to the first-order scattering of $E_{2 g}$ phonon from $\mathrm{sp}^{2}$-bonded carbon atoms constituting the graphite lattice, while the $\mathrm{D}$ mode is associated with structural imperfections and vibrations of carbon atoms with $\mathrm{sp}^{3}$ electronic configuration of C-O bonds $[36,37]$. The intensity ratio of the $D$ and $G$ bands $\left(I_{D} / I_{G}\right)$ is shown in the inset of Fig. 4. The ratio is a measure of disorder degree and is inversely proportional to the average size of the $\mathrm{sp}^{2}$ clusters [37]. As can be observed, the calculated $\mathrm{I}_{\mathrm{D}} / \mathrm{I}_{\mathrm{G}}$ ratio does not vary significantly in case of the samples irradiated with low, 20 and $40 \mathrm{~mJ} / \mathrm{cm}^{2}$ laser fluence values as compared to that corresponding to the reference sample. However, the further increase of the laser fluence to 60 and $80 \mathrm{~mJ} / \mathrm{cm}^{2}$ lead to the gradual diminishment of the $I_{D} / I_{G}$ ratio. This feature can be attributed to the decrease of the number of oxygen containing functional groups containing $\mathrm{sp}^{3}$ hybridised $\mathrm{C}$ atoms $[36,38]$ and formation of rGO at high laser fluence values.

As concerns anatase phase $\mathrm{TiO}_{2} \mathrm{NPs}$, no structural changes can be observed after direct laser processing at low, 20 and $40 \mathrm{~mJ} / \mathrm{cm}^{2}$ laser fluence values (Fig. 4a,b). However, the onset of anatase to rutile phase transition can be observed at higher laser fluencies (Fig. 4c,d). Besides the Raman bands assigned to anatase phase $\mathrm{TiO}_{2}$, the spectra of the $\mathrm{TiO}_{2} / \mathrm{GO}$ samples irradiated at 60 and $80 \mathrm{~mJ} / \mathrm{cm}^{2}$ laser fluences contain new bands at 436 and $612 \mathrm{~cm}^{-1}$, assigned to the Eg and A1g Raman vibrational modes of rutile phase $\mathrm{TiO}_{2}$ [39]. The intensity of the Raman bands increases with the increase of the laser fluence from 60 (Fig. 4c) to $80 \mathrm{~mJ} / \mathrm{cm}^{2}$ (Fig. 4d) under the accumulation of the laser pulses. As known, rutile is the thermodynamically stable phase of $\mathrm{TiO}_{2}$ and it can be obtained by thermal annealing of amorphous titanium oxide which transforms into anatase, while the bulk phase transition from anatase to rutile takes place in air at around $600{ }^{\circ} \mathrm{C}$ [1]. However, the transition temperatures reported in the literature vary in the range $400-1200{ }^{\circ} \mathrm{C}$ [40]. According to our FE-SEM 
investigations, the melting temperature is reached locally even at lower, $40 \mathrm{~mJ} / \mathrm{cm}^{2}$ laser fluence (Fig. 2b) surpassing the phase transition temperature. However, homogeneous surface melting takes place only at laser fluencies higher than $60 \mathrm{~mJ} / \mathrm{cm}^{2}$ (Fig. 2c). On the other hand, the position and width of the Raman bands belonging to $\mathrm{TiO}_{2} \mathrm{NPs}$ remain very similar for all samples indicating that the $[\mathrm{O}] /[\mathrm{Ti}]$ ratio is not changed under multipulse laser irradiation [39].

\section{Conclusions}

$\mathrm{TiO}_{2}$ / GO nanocomposite layers were deposited by spin-coating technique. The layers were submitted to direct laser irradiation using a frequency quadrupled Nd:YAG $(\lambda=266 \mathrm{~nm}$, $\tau_{\mathrm{FWHM}}=3 \mathrm{~ns}, v=10 \mathrm{~Hz}$ ) laser source. The effect of the incident laser fluence value as well as number of subsequent laser pulses on the surface morphology, crystalline structure, and chemical composition of the layers was investigated. Surpassing a laser fluence threshold value the $\mathrm{TiO}_{2}$ NPs were melted. The molten NPs were found to merge into inter-connected aggregates, leading to the formation of a network-like structure. Resonant ripple-like surface features formation on the surface of the GO platelets was observed at the highest laser fluence value. Moreover, direct laser irradiation lead to the decrease of the number of oxygen containing functional groups and formation of rGO as well as the onset of anatase to rutile phase transition at high fluence values. The stoichiometry of the $\mathrm{TiO}_{2} \mathrm{NPs}$ did not change under multipulse laser irradiation.

\section{Acknowledgments}

The authors acknowledge the financial support from the Romanian Ministry of Education and Scientific Research under the contract PNII-ID-PCE-2012-4-0292 and the Spanish Ministry of Economy and Competitiveness under the contract ENE2014-56109-C3-3-R. 
References

1. D. R. Lide, Handbook of Chemistry and Physics, 71st edition. CRC, Boca Raton, FL, 1991.

2. L. Sang, Y. Zhao, C. Burda, $\mathrm{TiO}_{2}$ Nanoparticles as Functional Building Blocks, Chem. Rev. 114 (2014) 9283-9318.

3. K. S. Novoselov, A. K. Geim, S. V. Morozov, D. Jiang, Y. Zhang, S. V. Dubonos, I. V. Grigorieva, A. A. Firsov, Electric Field Effect in Atomically Thin Carbon Films, Science, 306 (2004) 666-669.

4. A. H. Castro Neto, F. Guinea, N. M. R. Peres, K. S. Novoselov, A. K. Geim, Rev. Mod. Phys., 81 (2009) 109-162.

5. S. Chen, Q. Li, Q. Zhang, Y. Qu, H. Ji, R. S. Ruoff, W. Cai, Thermal conductivity measurements of suspended graphene with and without wrinkles by micro-Raman mapping, Nanotechnology 23 (2012) 365701 (4pp).

6. F. Bonaccorso, Z. Sun, T. Hasan, A. C. Ferrari, Graphene photonics and optoelectronics, Nature Photonics, 4 (2010) 611-622.

7. Y. Zhu, S. Murali, W. Cai, X. Li, J. W. Suk, J. R. Potts, R. S. Ruoff, Graphene and Graphene Oxide: Synthesis, Properties, and Applications, Adv. Mater. 22 (2010) 3906-3924.

8. C. Chung, Y. K. Kim, D. Shin, S. R. Ryoo, B. H. Hong, D. H. Min, Biomedical Applications of Graphene and Graphene Oxide, Acc. Chem. Res. 46 (2013) 22112224.

9. G. Wang, B. Wang, J. Park, J. Yang, X. Shen, J. Yao, Synthesis of enhanced hydrophilic and hydrophobic graphene oxide nanosheets by a solvothermal method, Carbon 47 (2009) 68-72.

10. J. W. Suk, R. D. Piner, J. An, R. S. Ruoff, Mechanical Properties of Monolayer Graphene Oxide, ACS Nano 4 (2010) 6557-6564.

11. L. Liu, J. Zhang, J. Zhao, F. Liu, Mechanical properties of graphene oxides, Nanoscale 4 (2012) 5910-5916.

12. S. Zhou, A. Bongiorno, Origin of the Chemical and Kinetic Stability of Graphene Oxide, Sci. Rep. 3 (2013) 2484 (7pp). 
13. D, R. Dreyer, S. Park, C. W. Bielawski, R. S. Ruoff, The chemistry of graphene oxide, Chem. Soc. Rev., 39 (2010) 228-240.

14. Y. Matsumoto, M. Koinuma, S. Ida, S. Hayami, T. Taniguchi, K. Hatakeyama, H. Tateishi, Y. Watanabe, S. Amano, Photoreaction of Graphene Oxide Nanosheets in Water, J. Phys. Chem. C 115 (2011) 19280-19286.

15. S. Stankovich, D. A. Dikin, R. D. Piner, K. A. Kohlhaas, A. Kleinhammes, Y. Jia, Y. Wu, S. B. T. Nguyen, R. S. Ruof, Synthesis of graphene-based nanosheets via chemical reduction of exfoliated graphite oxide, Carbon 45 (2007) 1558-1565.

16. H. J. Shin, K. K. Kim, A. Benayad, S. M. Yoon, H. K. Park, I. S. Jung, M. H. Jin, H. K. Jeong, J. M. Kim, J. Y. Choi, Y. H. Lee, Efficient Reduction of Graphite Oxide by Sodium Borohydride and Its Effect on Electrical Conductance, Adv. Funct. Mater. 19 (2009) 1987-1992.

17. C. Xiang, M. Li, M. Zhi, A. Manivannan, N. Wu, Reduced graphene oxide / titanium dioxide composites for supercapacitor electrodes: shape and coupling effects, J. Mater. Chem. 22 (2012) 19161-19167.

18. A. Sacco, S. Porro, A. Lamberti, M. Gerosa, M. Castellino, A. Chiodoni, S. Bianco, Investigation of Transport and Recombination Properties in Graphene/Titanium Dioxide Nanocomposite for Dye-Sensitized Solar Cell Photoanodes, Electrochim. Acta 131 (2014) 154-159.

19. W. K. Jo, H. J. Kang, Titanium dioxide-graphene oxide composites with different ratios supported by Pyrex tube for photocatalysis of toxic aromatic vapors, Powder Technol. 250 (2013) 115-121.

20. A. Datcu, L. Duta, A. Perez del Pino, C. Luculescu, C. Logofatu, A. Duta, D. Perniu, E. György, One-step preparation of nitrogen doped titanium oxide / Au / reduced graphene oxide composite thin films for photocatalytic applications, RSC Advances, 5 (2015) 49771-49779.

21. E. György, Á. Pérez del Pino, C. Logofatu, C. Cazan, A. Duta, Simultaneous laserinduced reduction and nitrogen doping of graphene oxide in titanium oxide/graphene oxide composites, J. Am. Ceram. Soc., 97 (2014) 2718-2724.

22. E. György, A. Perez del Pino, C. Logofatu, A. Duta, L. Isac, Effect of nitrogen doping on wetting and photoactive properties of laser processed zinc oxide-graphene oxide nanocomposite layers, J. Appl. Phys. 116 (2014) 024906 (7pp). 
23. O. Van Overschelde, T. Delsate, and R. Snyders, Determination of the melting threshold of $\mathrm{TiO}_{2}$ thin films processed by excimer laser irradiation, J. Appl. Phys. 111 (2012) 123108 (4pp).

24. E. György, I. N. Mihailescu, P. Serra, A. Perez del Pino, J. L. Morenza, Crown-like structure development on titanium exposed to multipulse Nd:YAG laser irradiation, Appl. Phys. A: Mater. Sci. \& Process. 74 (2002) 755-759.

25. S. Xiao, E. L. Gurevich, and A. Ostendorf, Incubation effect and its influence on laser patterning of ITO thin film, Appl. Phys. A. Mater. Sci. Process. 107 (2012) 333-338.

26. J. C. Miller, R. F. Haglund, Eds., Laser Ablation and Desorption, Academic Press, San Diego, CA, USA, 1998

27. C. Phipp, Ed., Laser Ablation and its Applications, Springer Scinece + Business Media, New York, NY, USA, 2007

28. D. Bäuerle, Laser Processing and Chemistry, Springer-Verlag, Heidelberg, Germany, 2011

29. A. Perez del Pino, E. György, C. Logofatu, J. Puigmartí-Luis, W. Gao, Laser-induced chemical transformation of graphene oxide-iron oxide nanoparticles composites deposited on polymer substrates, Carbon 93 (2015) 373-383.

30. A. Pérez del Pino, E. György, L. Cabana, B. Ballesteros, G. Tobias, Ultraviolet pulsed laser irradiation of multi-walled carbon nanotubes in nitrogen atmosphere, J. Appl. Phys., 115 (2014) 093501 (10 pp).

31. A. T. N'Diaye, R. van Gastel, A. J. Martínez-Galera, J. Coraux, H. Hattab, D. Wall, F. J. Meyer zu Heringdorf, M. Horn-von Hoegen, J. M. Gómez-Rodríguez, B. Poelsema, C. Busse, T. Michely, In situ observation of stress relaxation in epitaxial graphene, New J. Phys. 11 (2009) 113056 (14 pp).

32. C. G. Wang, L. Lan, Y. P. Liu, H. F. Tan, Defect-guided wrinkling in graphene, Comp. Mater. Sci. 77 (2013) 250-253.

33. C. Wang, Y. Liu, L. Li, H. Tan, Anisotropic thermal conductivity of graphene wrinkles. Nanoscale 6 (2014) 5703-5707.

34. F. Tian, Y. Zhang, J. Zhang, C. Pan, Raman Spectroscopy: A New Approach to Measure the Percentage of Anatase $\mathrm{TiO}_{2}$ Exposed (001) Facets, J. Phys. Chem. C 116 (2012) 7515-7519.

35. E. Silva Junior, F. A. La Porta, M. S. Liu, J. Andrés, J. A. Varela, E. Longo, A relationship between structural and electronic order-disorder effects and optical properties in crystalline $\mathrm{TiO}_{2}$ nanomaterials, Dalton Trans., 44 (2015) 3159-3175. 
36. K. N. Kudin, B. Ozbas, H. C. Schniepp, R. K. Prud'homme, I. A. Aksay, R. Car, Raman Spectra of Graphite Oxide and Functionalized Graphene Sheets, Nano Lett., 8 (2008) 36-41.

37. M. Cheng, R. Yang, L. Zhang, Z. Shi, W. Yang, D. Wang, G. Xie, D. Shi, G. Zhang, Restoration of graphene from graphene oxide by defect repair, Carbon, 50 (2012) 2581-2587.

38. O. Akhavan, E. Ghaderia, Escherichia coli bacteria reduce graphene oxide to bactericidal graphene in a selflimiting manner, Carbon 50 (2012) 1853-1860.

39. T. Mazza, E. Barborini, P. Piseri, P. Milani, D. Cattaneo, A. Li Bassi, C. E. Bottani, C. Ducati, Raman spectroscopy characterization of $\mathrm{TiO}_{2}$ rutile nanocrystals, Phys. Rev. B 75, (2007) 045416 (5 pp).

40. D. A. H. Hanaor, C. C. Sorrell, Review of the anatase to rutile phase transformation, J. Mater. Sci. 46 (2011) 855-874. 
Figure caption

Fig. 1. FE-SEM image of nanocomposite reference $\mathrm{TiO}_{2} / \mathrm{GO}$ nanocomposite thin film obtained by spin coating from $50 \mu \mathrm{l}$ dispersions consisting of 1 wt.\% $\mathrm{TiO}_{2}$ NPs and 0.5 wt. \% GO platelets.

Fig. 2. FE-SEM images of $\mathrm{TiO}_{2} / \mathrm{GO}$ nanocomposite thin films obtained by spin coating from $50 \mu \mathrm{l}$ dispersions consisting of $1 \mathrm{wt}$ \% $\mathrm{TiO}_{2}$ NPs and 0.5 wt. \% GO platelets irradiated in vacuum at a pressure of $10^{-4}$ Pa with 1000 laser pulses and (a) 20, (b) 40, (c) 60, and (d) 80 $\mathrm{mJ} / \mathrm{cm}^{2}$ laser fluence.

Fig. 3. FE-SEM images of $\mathrm{TiO}_{2} / \mathrm{GO}$ nanocomposite thin films obtained by spin coating from $50 \mu \mathrm{l}$ dispersions consisting of 1 wt.\% $\mathrm{TiO}_{2}$ NPs and 0.5 wt. \% GO platelets irradiated in vacuum at a pressure of $10^{-4}$ Pa with 100 laser pulses and (a) 60, as well as (b, c) $80 \mathrm{~mJ} / \mathrm{cm}^{2}$ laser fluence.

Fig. 4. Micro-Raman spectra of $\mathrm{TiO}_{2}$ / GO nanocomposite thin film obtained by spin coating from $50 \mu$ dispersions consisting of 1 wt.\% $\mathrm{TiO}_{2}$ NPs and 0.5 wt. \% GO platelets, (a) as deposited reference sample and (b-d) irradiated in vacuum at a pressure of $10^{-4} \mathrm{~Pa}$ with 1000 laser pulses and $20 \mathrm{~mJ} / \mathrm{cm}^{2}, 40 \mathrm{~mJ} / \mathrm{cm}^{2}, 60 \mathrm{~mJ} / \mathrm{cm}^{2}$ and $80 \mathrm{~mJ} / \mathrm{cm}^{2}$ laser fluence. The graph in the inset corresponds to the dependence of the $I_{D} / I_{G}$ intensity ratio of the $D$ and $G$ bands as a function of the laser fluence. 


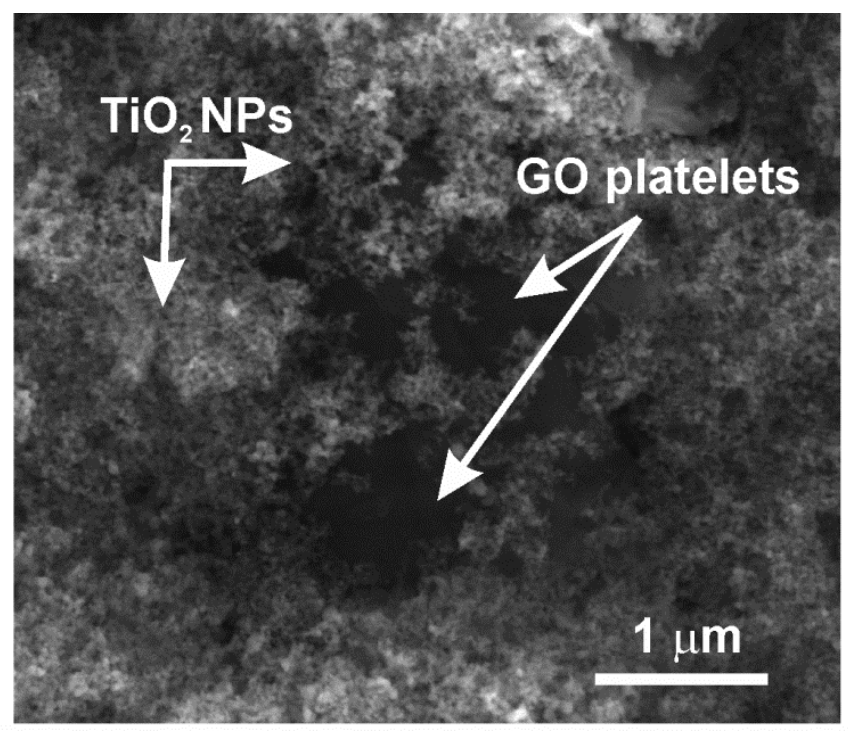

Fig. 1. 

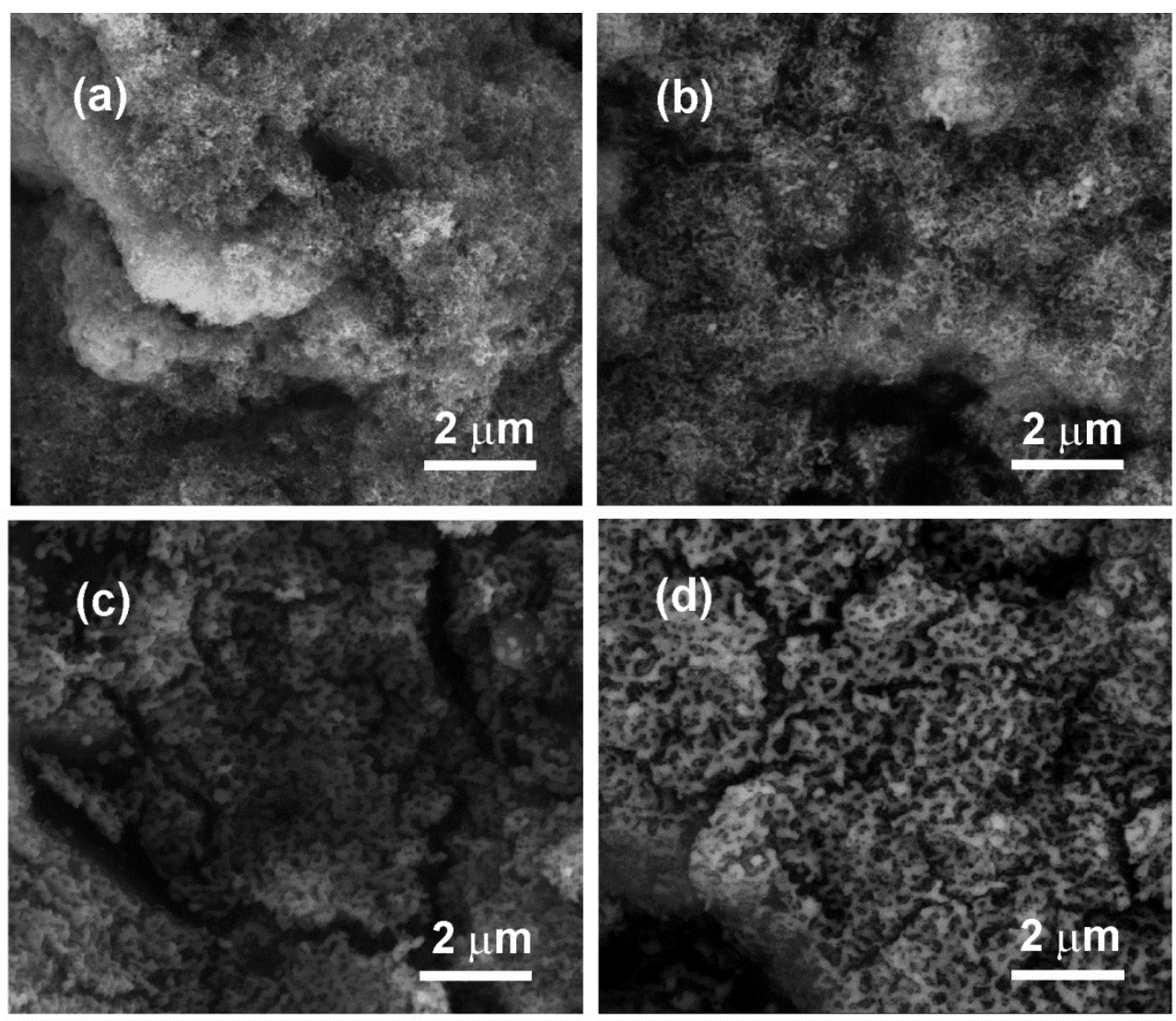

Fig. 2. 

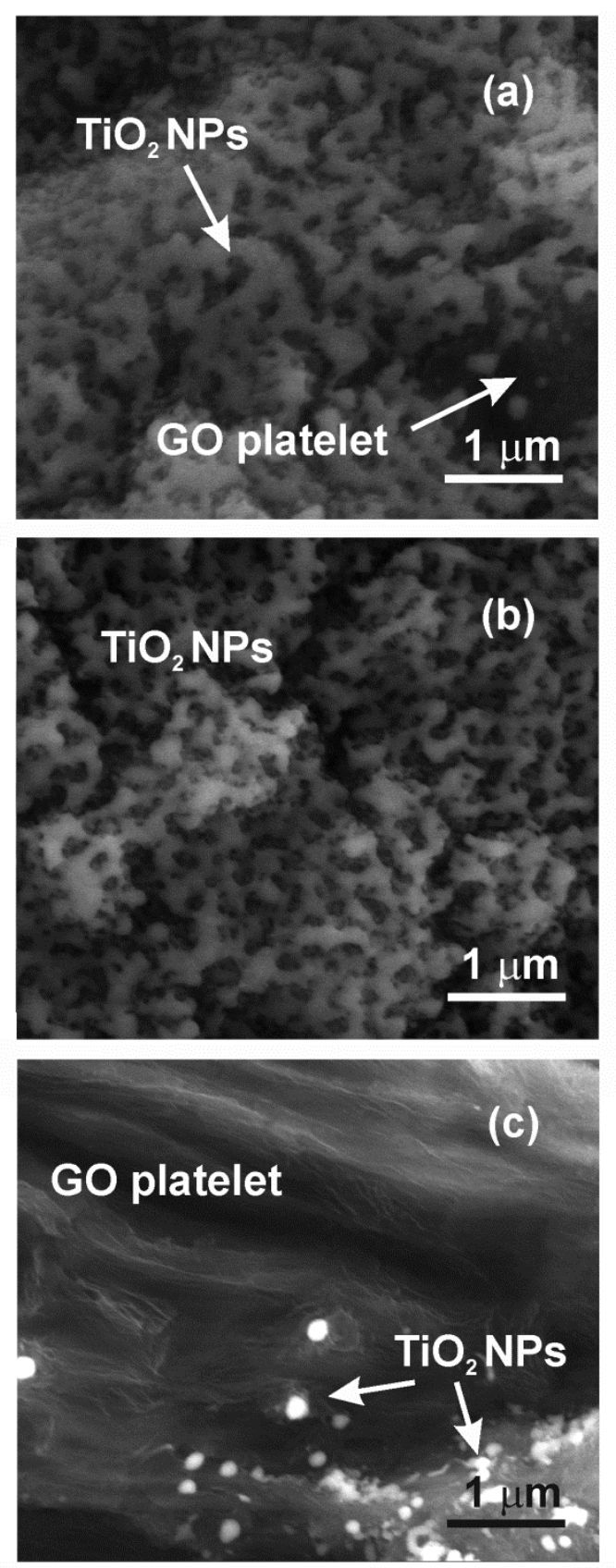

Fig. 3. 


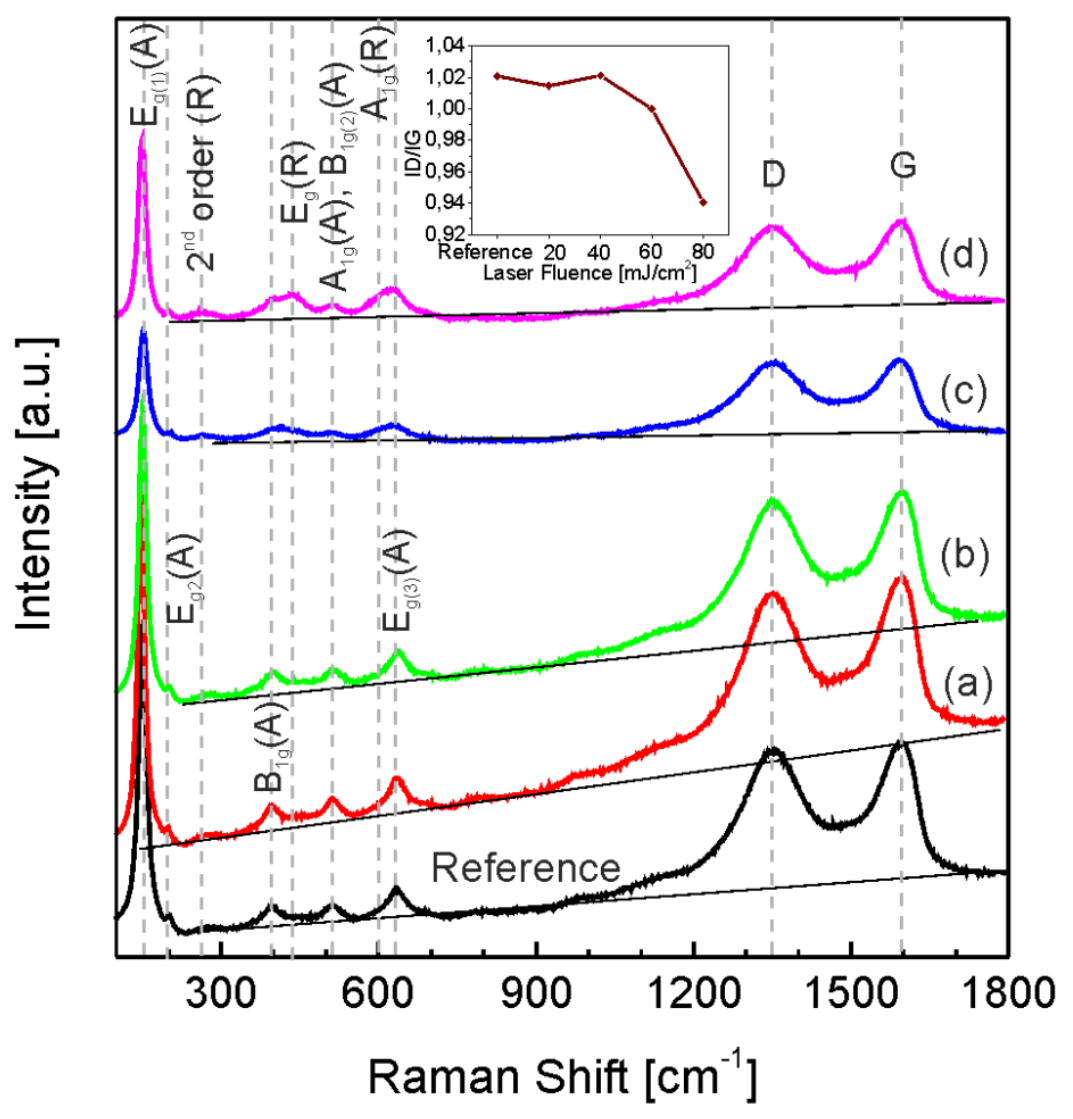

Fig. 4. 